\title{
Chemical conversion of human lung fibroblasts into neuronal cells
}

\author{
XIAO-YU WAN ${ }^{1 *}$, LI-YUN XU ${ }^{1 *},{\text { BING } \text { LI }^{1} \text {, QIU-HONG SUN }}^{1}$, QIU-LIANG JI ${ }^{1}$, \\ DONG-DONG HUANG ${ }^{1}$, LAN ZHAO $^{1}$ and YONG-TAO XIAO ${ }^{2}$ \\ ${ }^{1}$ Department of Respiratory Medicine, Shanghai Pulmonary Hospital, Tongji University School of Medicine, \\ Shanghai 200433; ${ }^{2}$ Shanghai Institute for Pediatric Research, Shanghai 200092, P.R. China
}

Received December 20, 2016; Accepted January 5, 2018

DOI: $10.3892 /$ ijmm.2018.3375

\begin{abstract}
It has been previously reported that human embryonic fibroblasts and mouse embryonic fibroblasts can be converted into neuronal cells using chemical agents, along with forced expression specific transcriptional factors. However, the materials required for reprogramming in these approaches presents major technical difficulties and safety concerns. The current study investigated whether a cocktail of small molecules can convert human lung fibroblast cells into neurons. The small molecules valproic acid, CHIR99021, DMH1, Repsox, forskolin, Y-27632 and SP600125 (VCHRFYS) were used to induce MRC-5 cells into neuronal cells in vitro. Neuronal markers were analyzed by immunofluorescence staining. The gene profiles were analyzed by reverse transcription-quantitative polymerase chain reaction. MRC-5 is a human lung fibroblast cell line derived from normal lung tissue of a 14-week-old male fetus. The results of the current study demonstrated that MRC-5 fibroblasts can be directly converted into neuronal cells using a cocktail of seven small molecules (VCHRFYS), with a yield of $~ 90 \%$ Tuj1-positive cells after 7 days of induction. Following a further maturation period, these chemically-induced neurons possessed neuronal morphology and expressed multiple neuron-specific genes. In conclusion, a cocktail of small molecules that can convert fibroblasts MRC-5 cells into functional neurons without the exogenous genetic factors was identified, which has the potential to be useful in neurological disease therapy.
\end{abstract}

\section{Introduction}

Since Takahashi and Yamanaka (1) demonstrated that a simple combination of four genes could reprogram somatic cells, direct

Correspondence to: Dr Yong-Tao Xiao, Shanghai Institute for Pediatric Research, Shanghai, P.R. China

E-mail: ytxiaocell@163.com

Dr Lan Zhao, Department of Respiratory Medicine, Shanghai Pulmonary Hospital, Tongji University School of Medicine, Shanghai 200433, P.R. China

E-mail: zhaolan72@163.com

*Contributed equally

Key words: fibroblast, neurons, small molecules, conversion, neurological disease lineage conversion has provided a rich source of somatic cell types for use in translational medicine. As the most commonly used somatic cells for direct conversion, fibroblasts can be directly converted into diverse functional cell types by introduction of known cell-fate-determining transcription factors or microRNAs (2-5). However, a major limitation related to current methods is the required ectopic expression of key developmental genes. Since the target genes often have to be stably integrated into the genome, the genetic modifications may have undesired effects. Chemical-based conversion strategies are gene-free, allowing the generation of cells without genetic modifications, and could be very tightly controlled.

Small molecules specifically modifying key signaling pathways provide a powerful tool to enhance conversion or even replace reprogramming genes. Indeed, a previous study showed that neural progenitor cells could be induced from mouse fibroblasts or human urinary cells with the appropriate chemical cocktail (6). Recently, chemical-promoted transdifferentiation from mouse embryonic fibroblasts or human foreskin fibroblasts to neuronal cells has been reported $(7,8)$. However, it is invasive and difficult to obtain these cells. The MRC-5 cell line was derived from the normal lung tissue of a 14-week-old male fetus (9). The aim of the current study was to convert MRC-5 cells into neuronal cells using a cocktail of small molecules.

\section{Materials and methods}

Materials and reagents. The information of the key reagents used in this study is presented in Table I. Small molecules and reagents included: Valproic acid (VPA; V); CHIR99021 (C); Repsox (R); forskolin (F); Y-27632 (Y); SP600125 (S); DMH1 (H); cyclic adenosine monophosphate (cAMP), $100 \mu \mathrm{M}$; brain-derived neurotrophic factor (BDNF), $20 \mathrm{ng} / \mathrm{ml}$; glial cell-derived neurotrophic factor (GDNF), $20 \mathrm{ng} / \mathrm{ml}$; neurotrophin-3 (NT3), $20 \mathrm{ng} / \mathrm{ml}$. Induction medium was composed of a 1:1 ratio of Dulbecco's modified Eagle's medium (DMEM)/F12 (cat. no. 11330032) and Neurobasal medium (cat. no. 21103049) with $0.5 \%$ N-2 supplement (cat. no. 17502048), 1\% B-27 supplement (cat. no. 17504044; all from Thermo Fisher Scientific, Inc., Waltham, MA, USA) and $100 \mu \mathrm{M}$ cAMP. Maturation medium was composed of DMEM/F12:Neurobasal medium (1:1), 0.5\% N-2 supplement, $1 \%$ B-27 supplement, $100 \mu \mathrm{M}$ cAMP, $20 \mathrm{ng} / \mathrm{ml} \mathrm{BDNF}$, $20 \mathrm{ng} / \mathrm{ml}$ GDNF and $20 \mathrm{ng} / \mathrm{ml}$ NT3. 
Table I. Reagents used in the current study.

\begin{tabular}{lll}
\hline Product code & \multicolumn{1}{c}{ Product name } & \multicolumn{1}{c}{ Manufacturer } \\
\hline R0158-5MG & Respox & Sigma-Aldrich; Merck KGaA (Darmstadt, Germany) \\
Y0503 & Y-27632 & Sigma-Aldrich; Merck KGaA \\
SML1046-5MG & CHIR99021 & Sigma-Aldrich; Merck KGaA \\
D8946-5MG & DMH1 & Sigma-Aldrich; Merck KGaA \\
F6886-10MG & Forskolin & Sigma-Aldrich; Merck KGaA \\
S7067-5MG & SB 202190 & Sigma-Aldrich; Merck KGaA \\
D0260-25MG & cAMP & Sigma-Aldrich; Merck KGaA \\
450-02-10 & Human BDNF & PeproTech, Inc. (Rocky Hill, NJ, USA) \\
$450-10-10$ & Human GDNF & PeproTech, Inc. \\
$450-03-10$ & Human NT-3 & PeproTech, Inc. \\
S5567 & SP600125 & Sigma-Aldrich (Merck KGaA) \\
PHR1061-1G & Valproic acid & Sigma-Aldrich (Merck KGaA) \\
845501 & Purified anti-tubulin 33 & BioLegend, Inc. (San Diego, CA, USA) \\
ab5392 & Anti-Map2 antibody & Abcam (Cambridge, UK) \\
ab177487 & Anti-NeuN antibody (EPR12763) neuronal marker & Abcam \\
ab80579 & Anti-Tau antibody (TAU-5) & Abcam \\
D9542 & DAPI & Sigma-Aldrich (Merck KGaA) \\
74104 & RNeasy mini kit (50) & Qiagen GmbH (Hilden, Germany) \\
33109 ES60 & Rhodamine (TRITC) AffiniPure & Jackson ImmunoResearch Laboratories, Inc. \\
& goat anti-rabbit IgG (H+L) & (West Grove, PA, USA) \\
$703-545-155$ & Alexa Fluor 488-AffiniPure & Jackson ImmunoResearch Laboratories, Inc. \\
& donkey anti-chicken IgY (IgG) (H+L) & \\
\hline
\end{tabular}

DMH1, 4-[6-(4-isopropoxyphenyl)pyrazolo[1,5-a]pyrimidin-3-yl]quinoline; cAMP, cyclic adenosine monophosphate; BDNF, brain-derived neurotrophic factor; GDNF, glial cell-derived neurotrophic factor; NT-3, neurotrophin-3; Map2, microtubule-associated protein 2; NeuN, RNA binding fox-1 homolog 3; TRITC, tetramethylrhodamine; Ig, immunoglobulin.

Table II. Primers for reverse transcription quantitative polymerase chain reaction.

\begin{tabular}{lll}
\hline Gene & \multicolumn{1}{c}{ Forward } & \multicolumn{1}{c}{ Reverse } \\
\hline Ascl1 & 5'-CAAGAGAGCGCAGCCTTAG-3' & 5'-GCAAAAGTCAGTGCTGAACG-3' \\
Brn2 & 5'-AATAAGGCAAAAGGAAAGCAACT-3' & 5'-CAAAACACATCATTACACCTGCT-3' \\
Myt11 & 5'-CAATGGAAAGGGATTTTAAGCA-3' & 5'-TTTGAGATTATGTACCAACGTTAGATG-3' \\
Ngn2 & 5'-TCAGACATGGACTATTGGCAG-3' & 5'-GGGACAGGAAAGGGAACC-3' \\
NeuroD1 & 5'-GTTATTGTGTTGCCTTAGCACTTC-3' & 5'-AGTGAAATGAATTGCTCAAATTGT-3' \\
Foxa2 & 5'-AGCAGAGCCCCAACAAGATG-3' & 5'-TCTGCCGGTAGAAGGGGAAGA-3' \\
Sox2 & 5'-CAAGATGCACAACTCGGAGA-3' & 5'-CGGGGCCGGTATTTATAATC-3' \\
Foxg1 & 5'-AGAAGAACGGCAAGTACGAGA-3' & 5'-TGTTGAGGGACAGATTGTGGC-3' \\
Pax6 & 5'-GGCAACCTACGCAAGATGGC-3' & 5'-TGAGGGCTGTGTCTGTTCGG-3' \\
Thy1 & 5'-ATCGCTCTCCTGCTAACAGTC-3' & 5'-CTCGTACTGGATGGGTGAACT-3' \\
Ctgf & 5'-CATCTCCACCCGGGTTACCAA-3' 1 '-AGTACGGATGCACTTTTTGC-3' \\
HPRT & 5'-GAGGGCCAAGACGAAGACATC-3' & 5'-CAGATCACGTCATCGCACAAC-3' \\
\hline
\end{tabular}

The primers were mostly referenced from a previous study (8). Ascl1, achaete-scute family bHLH transcription factor 1; Brn2, POU class 3 homeobox 2; Myt11, myelin transcription factor 1 like; Ngn2, neurogenin 2; NeuroD1, neuronal differentiation 1; Foxa2, forkhead box A2; Sox2, SRY-box 2; Foxg1, forkhead box G1; Pax6, paired box 6; Thy1, Thy-1 cell surface antigen; Ctgf, connective tissue growth factor; Col1a1, collagen type I $\alpha 1$ chain; HPRT, hypoxanthine phosphoribosyltransferase.

Generation of chemical induced-neurons. The MRC-5 cells (American Type Culture Collection, Manassas, VA, USA) were seeded onto 6-well culture plates and cultured in Eagle's minimum essential medium (Gibco, Carlsbad, CA, USA) 


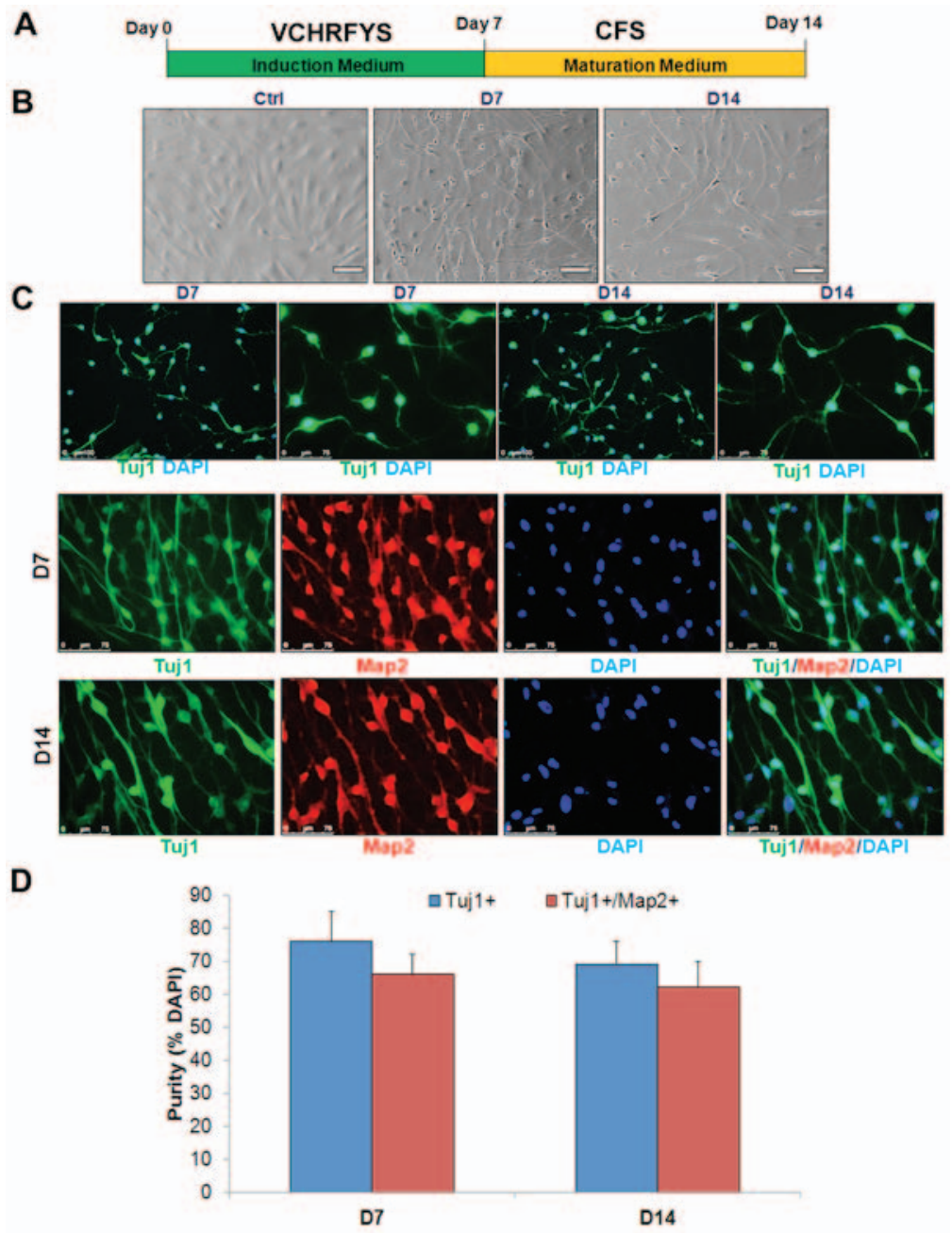

Figure 1. Generation of human neuronal cells by small molecules. (A) Scheme of induction procedure. (B) The cells display bipolar neuronal morphologies after 7 days introduction and 7 days maturation. Scale bars, $100 \mu \mathrm{m}$. (C) The chemicals induced-cells display express Tuj1 (green) and Tuj1 $1^{+} / \mathrm{Map}^{+}$. Scale bars, $75 \mathrm{~mm}$. (D) The percentage of induced Tuj1 ${ }^{+}$and Tuj1 $1^{+} / \mathrm{Map}^{+}$. Neuronal cells in total cells at the time of quantification (means \pm SD, $n=10$ random selected 50 fields from triplicate samples). V, VPA; C, CHIR99021; R, Repsox; F, forskolin; S, SP600625; Y, Y-27632; H, DMH1; S, SP600125; D, day; Tuj1, tubulin $\beta 3$ class III; Map2, microtubule-associated protein 2.

containing $10 \%$ fetal bovine serum (Gibco) at $37^{\circ} \mathrm{C}$ with $5 \% \mathrm{CO}_{2}$ in a humidified atmosphere for $48 \mathrm{~h}$. The induction medium with the chemical cocktail VCHRFYS was used to induce these primary cells when the cell confluence was $50-70 \%$. The concentration of chemicals used was as follows: VPA, $1 \mathrm{mM}$; CHIR99021, $3 \mu \mathrm{M}$; R, Repsox, $1 \mu \mathrm{M}$; forskolin, $10 \mu \mathrm{M}$; Y-27632, $5 \mu \mathrm{M}$; SP600125, $10 \mu \mathrm{M}$; DAPT, $5 \mu \mathrm{M}$ and DMH1, $2 \mu \mathrm{M}$. Medium containing chemical compounds was half-changed every three days. After 5 days, cells were switched to maturation medium with CFS (CHIR99021, $3 \mu \mathrm{M}$; forskolin, $10 \mu \mathrm{M}$; and SP600125, $10 \mu \mathrm{M}$ ) for 7 days. Maturation medium was half-changed every 2 day. Conversion efficiency was calculated as previously described $(4,8)$. Briefly, 10 fields of view were randomly selected for each sample on a Nikon Ti-E microscope (Nikon Corporation, Tokyo, Japan) at indicated time points, and the total cell number of tubulin $\beta 3$ class III (Tuj1), Tuj $1^{+} /$ microtubule-associated protein 2 (Map2) ${ }^{+}, \mathrm{Map2}^{+} /$RNA binding fox-1 homolog $3(\mathrm{NeuN})^{+}$, or $\mathrm{Tuj}^{+} / \mathrm{Tau}^{+}$cells with neuron morphology were counted following immunofluorescence staining. The conversion efficiency was calculated as the ratio of Tuj1, Tuj1 ${ }^{+} / \mathrm{Map2}^{+}, \mathrm{Map2}^{+} / \mathrm{NeuN}^{+}$, or Tuj1 ${ }^{+} / \mathrm{Tau}^{+}$cells to the initial seeding cells in each visual field. The purity represented the percentage of induced Tuj1, Tuj1 $1^{+} \mathrm{Map}^{+}, \mathrm{Map}^{+} / \mathrm{NeuN}^{+}$, or $\mathrm{Tuj}^{+} / \mathrm{Tau}^{+}$neuronal cells in the total number of cells stained with DAPI. Quantitative data are presented as the mean \pm standard error of at least three independent experiments.

Immunofluorescence staining. Immunostaining of cells were performed as previously described (10). Briefly, cells were washed with PBS twice and then fixed with $4 \%$ paraformaldehyde for $10 \mathrm{~min}$ at room temperature. Then cells were blocked with 5\% bovine serum albumin (MP Biomedicals, Auckland, New Zealand) containing $0.5 \%$ Triton X-100 for $30 \mathrm{~min}$ at room temperature. Subsequently, samples were incubated with primary antibodies (dilutions $1: 100$ ) at $4^{\circ} \mathrm{C}$ overnight and then with appropriate fluorescent probe-conjugated secondary antibodies for $1 \mathrm{~h}$ at room temperature. Nuclei were counterstained with DAPI. Images were captured using a fluorescence microscope (DM6000; Leica Microsystems GmbH, Wetzlar, Germany). The primary antibodies were used at the following dilutions: Tuj1, 1:100; Map2, 1:100; NeuN, 1:50; and Tau, 1:200. 


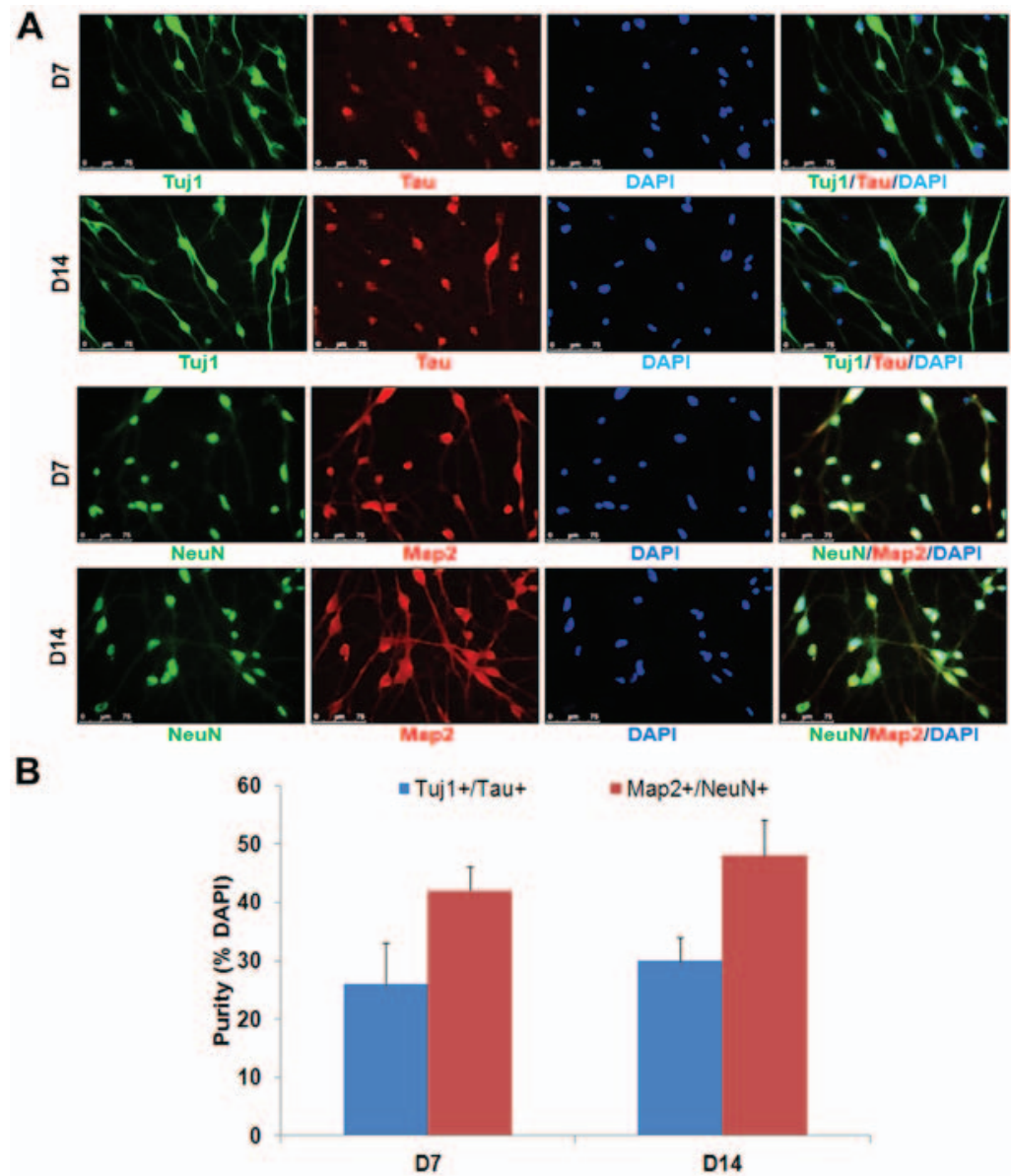

Figure 2. Cell phenotype of small molecule-induced cells. (A) Induced cells expressed Tuj1, Map2, Tau and NeuN. Scale bar, $75 \mu \mathrm{m}$. (B) Percentage of induced $\mathrm{Tujl}^{+} / \mathrm{Tau}^{+}$and $\mathrm{Map}^{+} / \mathrm{NeuN}^{+}$neuronal cells in total cells at the time of quantification (mean \pm standard error, $\mathrm{n}=10$ random selected 50 fields from triplicate samples). D, day; Tuj1, tubulin $\beta 3$ class III; NeuN, RNA binding fox-1 homolog 3; Map2, microtubule-associated protein 2.

Reverse transcription-quantitative polymerase chain reaction $(R T-q P C R)$. RNA was extracted with the RNeasy mini kit (cat. no. 74104; Qiagen GmbH Hilden, Germany) according to the manufacturer's protocol. For each reaction, $1 \mu \mathrm{g}$ RNA was reverse-transcribed to cDNA using a High Capacity cDNA RT kit (Applied Biosystems; Thermo Fisher Scientific, Inc.). qPCR was subsequently conducted with specific primers and SYBR-Green Universal Master Mix kit (Applied Biosystems; Thermo Fisher Scientific, Inc.) in a Pikoreal PCR machine (Thermo Fisher Scientific, Inc.). The relative expression levels were normalized against hypoxanthine phosphoribosyltransferase as the internal control. All reactions were repeated three times. The primers used are listed in Table II.

Statistical analysis. All quantified data were statistically analyzed and presented as the mean \pm standard error. For comparisons between two groups, a Student's t-test was used. For multiple group comparisons, one-way analysis of variance (ANOVA) was used to calculate statistical significance followed by Fisher's post hoc test. $\mathrm{P}<0.05$ was considered to indicate a statistically significant difference.

\section{Results}

Generation of neuronal cells from human MRC-5 lung fibroblasts using small molecules. Previous studies reported that a combination of small chemicals, VCRF, had important effects on the neural differentiation of mouse and human somatic cells, and neuronal cell survival (6-8). We hypothesized that the combination of VCRF may facilitate the conversion of human MRC-5 lung fibroblast cells into neuronal cells. The small molecules Y-27632 [Rho-associated, coiled-coil containing protein kinase (ROCK) inhibitor; Y) and SP600125 [c-Jun N-terminal kinase (JNK) inhibitor; S) were previously reported to promote neuronal conversion $(11,12)$. It was reported that DMH1, a highly selective small molecule bone morphogenic protein (BMP) inhibitor, has been demonstrated to promote neurogenesis of human-induced pluripotent stem cells (13). Thus, in the current study, MRC-5 cells were treated with a neuronal induction medium containing VCHRFYS for 7 days (Fig. 1A). VCHRFYS treatment induced a large fraction of the live cells to exhibit typical neuronal morphology, and expression of neuronal markers, including Tuj1, Map2, Tau and NeuN (Figs. 1B-D and 2). It was observed that the majority of the induced cells survived until day 8-10, and failed to become more mature neurons. The CFS chemical cocktail was then used to promote neuron survival and maturation. Indeed, neuronal cell survival and maturation were significantly improved after replacing the induction medium with the neuronal maturation medium supplemented with CFS and extra neurotropic factors (BDNF, GDNF and NT3). After 7 days of maturation, the cells were stained positive for neuronal markers Tau and NeuN (Fig. 2). 


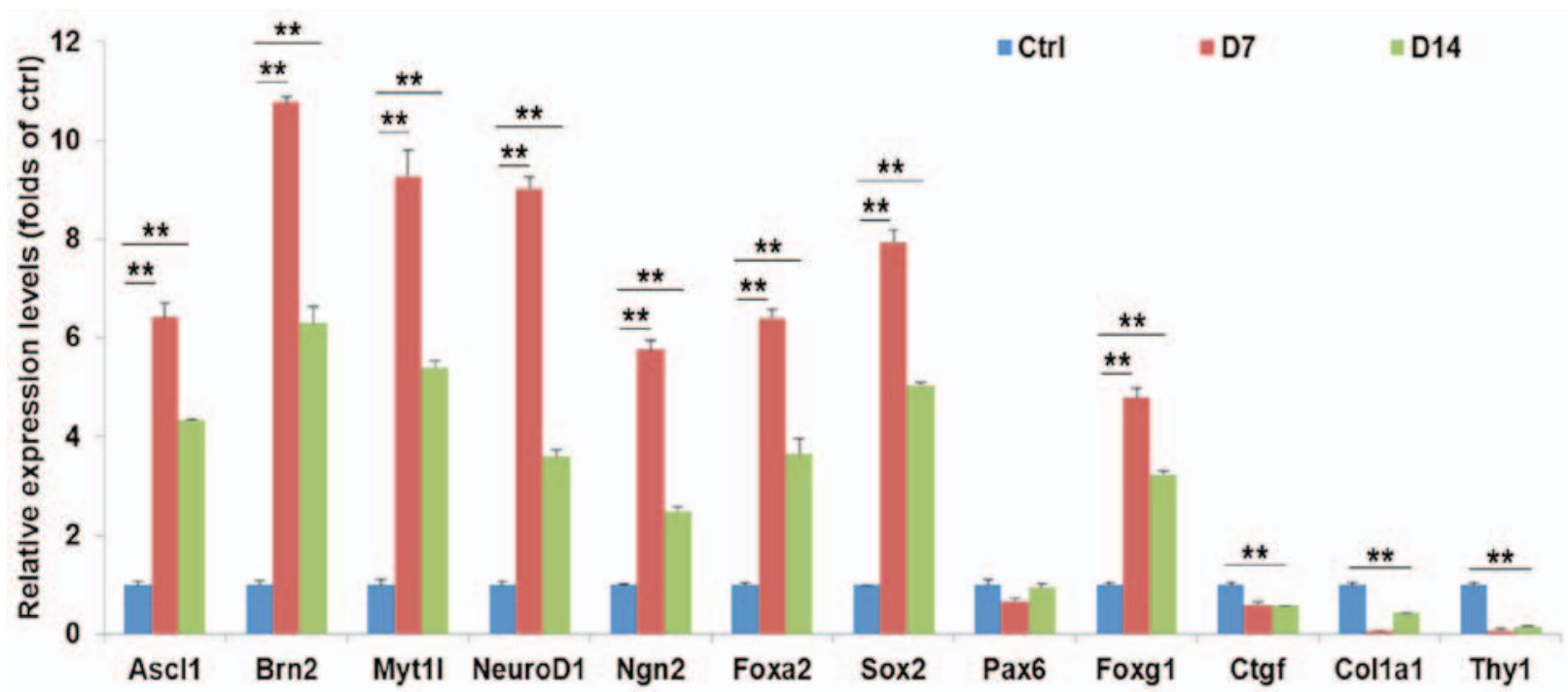

Figure 3. Genes profiles from small molecule-induced cells. The master neuronal genes were upregulated, while the genes associated with fibroblasts were downregulated in the small molecule-induced cells. The data are presented as the mean \pm standard error. Ascl1, achaete-scute family bHLH transcription factor 1; Brn2, POU class 3 homeobox 2; Myt11, myelin transcription factor 1 like; NeuroD1, neuronal differentiation 1; Ngn2, neurogenin 2; Foxa2, forkhead box A2; Sox2, SRY-box 2; Pax6, paired box 6; Foxg1, forkhead box G1; Ctgf, connective tissue growth factor; Colla1, collagen type I $\alpha 1$ chain; Thy1, Thy-1 cell surface antigen.

Table III. Small molecule compounds used in this study reported to affect neural cells reprogramming.

\begin{tabular}{|c|c|c|}
\hline Small molecules & Effects on neural cells conversion & Refs. \\
\hline $\begin{array}{l}\text { Valproic acid, } \\
\text { HDAC inhibitors }\end{array}$ & $\begin{array}{l}\text { Promote neurons differentiation and survival } \\
\text { of neural progenitor cells }\end{array}$ & $(14,15)$ \\
\hline CHIR99021 & Improve conversion of human into neurons and induce & \\
\hline GSK-3 inhibitor & pluripotent stem cells into nociceptors & $(16,17)$ \\
\hline Repsox & Improve generation of neural progenitor cells & $(6)$ \\
\hline TGF- $\beta$ inhibitor & from pluripotent stem cells & \\
\hline Forskolin & Promote neuronal conversion of human fibroblasts & \\
\hline cAMP activator & & $(18,19)$ \\
\hline $\begin{array}{l}\text { Y-27632, } \\
\text { ROCK inhibitor }\end{array}$ & Induce neural crest-like cells derived from human neural progenitors & $(11)$ \\
\hline $\begin{array}{l}\text { SP600125 } \\
\text { JNK inhibitor }\end{array}$ & Increases survival of transplanted dopamine neurons in Parkinsonian rats & (12) \\
\hline DMH1, BMP inhibitor & Increases neurogenesis of human-induced pluripotent stem cells & (13) \\
\hline
\end{tabular}

HDAC, histone deacelylase; GSK-3, glycogen synthase kinase-3; cAMP, cyclic adenosine monophosphate; TGF- $\beta$, transforming growth factor- $\beta$; ROCK, Rho-associated, coiled-coil containing protein kinase; JNK, c-Jun N-terminal kinase; BMP, bone morphogenetic protein.

Chemical-induced cells exhibit a neuronal gene expression pattern. To further characterize the induced neuronal cells phenotype, the expression of nine neuronal genes and three fibroblastic genes were quantitatively analyzed using RT-qPCR. Several representative neuronal-specific genes, including achaete-scute family bHLH transcription factor 1 , POU class 3 homeobox 2, myelin transcription factor 1 like and neuronal differentiation 1 , were significantly upregulated in VCHRFYS-treated MRC-5 cells on days 7 (Fig. 3). Notably, these neuronal markers slightly decreased during the process of maturation under treatment with CFS chemicals. By contrast, fibroblasts-specific genes, including collagen type I $\alpha 1$ chain, Thy- 1 cell surface antigen and connective tissue growth factor, were significant downregulated during neuronal conversion (Fig. 3). Taken together, the results demonstrated that the VCHRFYS chemical cocktail reduces fibroblast-specific gene expression in MRC-5 cells, specifically upregulating neuronal gene expression and facilitating the neuronal conversion of human lung fibroblasts. However, the precise regulatory mechanisms of this chemical cocktail remain to be investigated.

\section{Discussion}

Direct lineage conversion provides a rich source of somatic cell types for use in regenerative medicine. Direct conversion 
of non-neural cells to functional neurons is a promising strategy for cell-based therapy in the treatment of neurological disease. In recent years, several studies have demonstrated that somatic cells, and even terminally differentiated cells, could be reprogrammed into other cell types. It has been recently reported that ectopic expression of miR-9/9* and miR-124 (miR-9/9*-124) could promote direct conversion of human fibroblasts into neurons using viral vectors $(5,20)$. However, they may have effects on genetic modifications.

A previous study showed that a chemical cocktail VCR could induce neural progenitor cells from mouse embryonic fibroblasts and human urinary cells under a hypoxic condition (6). Another report showed that chemical VCRFSGY could convert the fibroblasts from human foreskin or skin into neuronal cells quickly (8). In the present approach, generation of neuronal cells from MRC-5 cells was induced by sequential treatment with defined compounds (VCHRFYS) (Table III). Histone deacetylase inhibitor, VPA, treatment induces cells into a more amenable state for cell-fate transition through epigenetic modification (21). CHIR99021 (glycogen synthase kinase-3 $\beta$ inhibitor), Repsox (transforming growth factor- $\beta$ inhibitor) and DMH1 (BMP inhibitor) improved the neuronal conversion of human fibroblasts (22). Forskolin (ROCK2 inhibitor) promotes migration of neural crest cells, and enables neurogenin 2 to convert human fibroblasts into cholinergic neurons (19). Y-27632 (Rho kinase inhibitor) enhances the maintenance of pluripotent stem cells and neuron survival (23). SP600125 (JNK inhibitor) can facilitate the neuronal conversion of fibroblasts (24). Thus, the chemical cocktail VCHRFYS reduces fibroblast-specific gene expression of the exposed cells, specifically inducing neuronal gene expression and facilitating the neuronal conversion of MRC-5 lung fibroblasts. However, the precise regulatory mechanisms of this chemical cocktail remain to be investigated. Taken together, the results of the current study demonstrated that MRC-5, a human lung fibroblast cell line, can be directly converted into neuronal cells by small compounds, which does not depend on ectopic gene expression, it is based on chemical modification of defined signaling pathways, such as TGF- $\beta$ and SMAD signaling $(25,26)$.

\section{Acknowledgements}

This study is supported by the National Natural Science Foundation of China (grant no. 81400861). We would like to thank B.L.G., Y.N.X., P.W. and C.Q.CH for their technical supports.

\section{Competing interests}

The authors declare that they have no competing interests.

\section{References}

1. Takahashi K and Yamanaka S: Induction of pluripotent stem cells from mouse embryonic and adult fibroblast cultures by defined factors. Cell 126: 663-676, 2006.

2. Xu J, Du Y and Deng H: Direct lineage reprogramming: Strategies, mechanisms, and applications. Cell Stem Cell 16: 119-134, 2015.

3. Yashar M, Kalani S and Martirosyan N: Direct conversion of fibroblasts to functional neurons. World Neurosurg 77: 7-8, 2012.

4. Vierbuchen T, Ostermeier A, Pang ZP, Kokubu Y, Südhof TC and Wernig M: Direct conversion of fibroblasts to functional neurons by defined factors. Nature 463: 1035-1041, 2010.
5. Yoo AS, Sun AX, Li L, Shcheglovitov A, Portmann T, Li Y, Lee-Messer C, Dolmetsch RE, Tsien RW and Crabtree GR: MicroRNA-mediated conversion of human fibroblasts to neurons. Nature 476: 228-231, 2011.

6. Cheng L, Hu W, Qiu B, Zhao J, Yu Y, Guan W, Wang M, Yang W and Pei G: Generation of neural progenitor cells by chemical cocktails and hypoxia. Cell Res 25: 645-646, 2015.

7. Li X, Zuo X, Jing J, Ma Y, Wang J, Liu D, Zhu J, Du X, Xiong L, Du Y, et al: Small-molecule-driven direct reprogramming of mouse fibroblasts into functional neurons. Cell Stem Cell 17: 195-203, 2015.

8. Hu W, Qiu B, Guan W, Wang Q, Wang M, Li W, Gao L, Shen L, Huang Y, Xie G, et al: Direct Conversion of normal and Alzheimer's disease human fibroblasts into neuronal cells by small molecules. Cell Stem Cell 17: 204-212, 2015.

9. Jacobs JP, Jones CM and Baille JP: Characteristics of a human diploid cell designated MRC-5. Nature 227: 168-170, 1970.

10. Xiao Y, Wang J, Yan W, Zhou Y, Chen Y, Zhou K, Wen J, Wang Y and Cai W: Dysregulated miR-124 and miR-200 expression contribute to cholangiocyte proliferation in the cholestatic liver by targeting IL-6/STAT3 signalling. J Hepatol 62: 889-896, 2015.

11. Hotta R, Pepdjonovic L, Anderson RB, Zhang D, Bergner AJ, Leung J, Pébay A, Young HM, Newgreen DF and Dottori M: Small-molecule induction of neural crest-like cells derived from human neural progenitors. Stem Cells 27: 2896-2905, 2009.

12. Rawal N, Parish C, Castelo-Branco G and Arenas E: Inhibition of JNK increases survival of transplanted dopamine neurons in Parkinsonian rats. Cell Death Differ 14: 381-383, 2007.

13. Neely MD, Litt MJ, Tidball AM, et al.: DMH1, a highly selective small molecule BMP inhibitor promotes neurogenesis of hiPSCs: comparison of PAX6 and SOX1 expression during neural induction. ACS Chem Neurosci. 3(6):482-91, 2012.

14. Kim BW, Yang S, Lee CH and Son H: A critical time window for the survival of neural progenitor cells by HDAC inhibitors in the hippocampus. Mol Cells 31: 159-164, 2011.

15. Jeong SG, Ohn T, Kim SH and Cho GW: Valproic acid promotes neuronal differentiation by induction of neuroprogenitors in human bone-marrow mesenchymal stromal cells. Neurosci Lett 554: 22-27, 2013.

16. Lu J, Liu H, Huang CT, Chen H, Du Z, Liu Y, Sherafat MA and Zhang SC: Generation of integration-free and region-specific neural progenitors from primate fibroblasts. Cell Rep 3: 1580-1591, 2013.

17. Chambers SM, Qi Y, Mica Y, Lee G, Zhang XJ, Niu L, Bilsland J, Cao L, Stevens E, Whiting P, et al: Combined small-molecule inhibition accelerates developmental timing and converts human pluripotent stem cells into nociceptors. Nat Biotechnol 30: 715-720, 2012.

18. Babos K and Ichida JK: Small molecules take a big step by converting fibroblasts into neurons. Cell Stem Cell 17: 127-129, 2015.

19. Liu ML, Zang T, Zou Y, Chang JC, Gibson JR, Huber KM and Zhang CL: Small molecules enable neurogenin 2 to efficiently convert human fibroblasts into cholinergic neurons. Nat Commun 4: 2183, 2013.

20. Victor MB, Richner M, Hermanstyne TO, et al.: Generation of human striatal neurons by microRNA-dependent direct conversion of fibroblasts. Neuron 84(2):311-23, 2014.

21. Huangfu D, Maehr R, Guo W, et al.: Induction of pluripotent stem cells by defined factors is greatly improved by small-molecule compounds. Nat Biotechnol. 26(7):795-7, 2008.

22. Ladewig J, Mertens J, Kesavan J, et al:: Small molecules enable highly efficient neuronal conversion of human fibroblasts. Nat Methods. (6):575-8, 2012.

23. Xi G, Hu P, Qu C, et al.: Induced neural stem cells generated from rat fibroblasts. Genomics Proteomics Bioinformatics. 11(5):312-9, 2014.

24. Zhu S, Ambasudhan R, Sun W, et al.: Small molecules enable OCT4-mediated direct reprogramming into expandable human neural stem cells. Cell Res. 24(1):126-9, 2014.

25. Ichida JK, Blanchard J, Lam K, et al.: A small-molecule inhibitor of tgf-Beta signaling replaces sox 2 in reprogramming by inducing nanog. Cell Stem Cell 5:491-503, 2009.

26. Chambers SM., FasanobCA., Papapetrou EP, et al.: Highly efficient neural conversion of human ES and iPS cells by dual inhibition of SMAD signaling. Nat Biotechnol 27:275-280, 2009.

This work is licensed under a Creative Commons Attribution-NonCommercial-NoDerivatives 4.0 International (CC BY-NC-ND 4.0) License. 